\title{
Desempeño de la Biomasa Microalgal para la microproducción de Biodiesel a partir de una brecha de potencial energético
}

Performance of the Microalgal Biomass for the microdiesel production from a gap of energy potential

O. Medina-Carpio, E. Medina-Cabrera, Janet Medina-Pérez, P. Manrique-Pino, P. Macedo-Benavente y J. Villanueva-Salas.

Universidad Católica de Santa María. Arequipa Perú.

\section{INFORMACIÓN}

\section{Historia del Artículo \\ Recepción: 18/02/2019 \\ Revisión: 22/04/2019}

Aceptación: 25/04/2019

\section{Palabras Clave}

Potencial energético, biomasa, microproductores, biodiesel y Chlorella sp.

\section{Key Words}

Energy potential, biomass, microproducers, biodiesel and Chlorella sp

\section{DOI}

https://doi.org/10.35286/veritas. v20i1.229

\begin{abstract}
RESUMEN
Se identificó que el potencial energético (PE) del biodiesel generado por microproductores en Arequipa es de 8042,2495 MJ*Kg-1 obtenido a partir de 50 gal de biodiesel (B100), por tal razón el presente trabajo de investigación tuvo como objetivo determinar las implicancias técnicas para alcanzar las brechas principales que deben superar los esfuerzos en investigación y desarrollo (I+D) para alcanzar el PE deseado utilizando biomasa microalgal como principal fuente de aceite transesterificado, la investigación demostró que es posible obtener $0,82 \mathrm{~g} / \mathrm{L}$ de biomasa microalgal a partir de Chlorella sp. en un sistema Raceway logrando extraer $614,90 \mathrm{~g} / \mathrm{kg}$ de aceite a partir de biomasa microalgal seca alcanzando a generar 554,32 g/Kg de aceite transesterificado (Biodiesel-B100), para realizar la investigación se utilizó como modelo a la microempresa ALDECO. Los resultados determinaron que para un microproductor que busca alcanzar un potencial energético (PE) de 8042,2495 MJ Kg-1 a partir de un potencial de producción factible (PPF) de $50 \mathrm{gal} / \mathrm{mes}$ que implica destinar un área de 2,35 ha bajo un rendimiento determinado por el potencial de biomasa disponible (PBD) de $820 \mathrm{~g} / \mathrm{m} 3$ y de combustible generado (PCG) de $28,55 \mathrm{~g} / \mathrm{m} 3$ en medio de cultivo BBM.
\end{abstract}

\begin{abstract}
It was identified the energy potential (PE) of biodiesel generated by microproducers in Arequipa is $8042.2495 \mathrm{MJ} * \mathrm{Kg}-1$ obtained from 50 gallons of biodiesel (B100), for this reason the present research work aimed at the implications techniques to reach the main gaps that must be overcome in research and development (R \& D) to achieve the desired PE in the microalgal biomass as the main source of transesterified oil, the research showed that it is possible to obtain $0.82 \mathrm{~g} / 1$ Biomass microalgal from Chlorella sp. in a Raceway system managing to extract $614.90 \mathrm{~g} / \mathrm{kg}$ of oil from dry microalgal biomass at your reach $554.32 \mathrm{~g} / \mathrm{Kg}$ of transesterified oil (Biodiesel-B100), to carry out an investigation as a model for the ALDECO microenterprise. The results were determined for a microproducer looking for an energy potential (PE) of 8042.2495 MJ Kg-1 with a feasible production potential (PPP) of $50 \mathrm{gal} /$ month implying an area of 2.35 ha under a yield determined by the available biomass potential (PBD) of $820 \mathrm{~g} / \mathrm{m} 3$ and of generated fuel (PCG) of $28.55 \mathrm{~g} / \mathrm{m} 3$ in BBM culture medium.
\end{abstract}

\section{INTRODUCCIÓN}

En los últimos años, la ruta de conversión de microalgas a combustible biodiesel ha sido ampliamente explorada. Hay un cambio de combustible a base de petróleo a biodiesel porque este último no es tóxico, es renovable y biodegradable, su combustión es más limpia ofreciendo una mayor lubricidad y propiedades de alto punto de inflamación (de Luna, Doliente, Ido, \& Chung,, 2017). Es por ello, que la presente investigación busca identificar el potencial de biomasa disponible y de combustible generado (PBD y PCG) en un sistema Race Way a condiciones ambientales debido a que los cultivos fotoautotróficos de microalgas comúnmente se realizan de manera convencional utilizando $\mathrm{CO} 2$ como fuente de carbono. Sin embargo, los procesos relacionados con la purificación, almacenamiento y distribución de $\mathrm{CO} 2$

Correspondencia:

O. Medina-Carpio

omedina@biotcorp.com aumentan el costo de producción de las microalgas, esta tecnología involucra una opción no competitiva para la producción de biocombustibles (Hanifzadeh, Sarrafzadeh, M. H., Nabati Z, Tavakoli, \& Feyzizarnagh, 2018). Por tal motivo, identificar el desempeño de la biomasa microalgal en condiciones ambiental da un indicador del potencial local para el desarrollo de la tecnología del biocombustible en estudio.

La producción de biodiesel a partir de microalgas suele involucrar cuatro etapas, incluyendo el cultivo de algas, la cosecha, la extracción de aceite y transesterificación (Torres, y otros, 2013), la investigación busca en la etapa de extracción de aceite identificar el desempeño de las microalgas heterotróficas capaces de acumular lípidos para que en posteriores investigaciones se busque evaluar las tecnologías que puedan superar brechas altas (hasta 57\% p / p) (Zhang, Yan, Tyagi, \& Surampalli, 2013), las microalgas acumulan estos lípidos en diversas condiciones de estrés (como limitación de nutrientes, baja intensidad de luz, alta salinidad, etc.) en diferentes formas que incluyen triglicéridos, ácidos grasos libres, esteroles, ésteres de cera, etc que pueden 
constituir hasta el $60 \%$ del peso de las células secas en ciertas especies (Ghosh, Roy, \& Das, 2015), en la investigación se procuró evaluar el contenido de aceites para identificar la microalga con mejor adaptabilidad en el medio y producción de lipídica, del mismo modo en la investigación se consideró que simulaciones realizadas mediante una herramienta específica para el diseño habilitado en el software Aspen Plus 2006.5TM identificó una brecha aproximada de 450,000 1/ día de biodiesel desde 17,300 kg/día de aceite de microalgal (Sánchez, Ojeda, El-Halwagi, \& Kafarov, 2011).

En la etapa de transesterificación, comúnmente el aceite extraído y refinado a partir de biomasa de microalgas se convierte en ésteres metílicos de ácidos grasos (FAME) por alcalinos o la transesterificación ácida. Este proceso ha sido ampliamente estudiado en la literatura (Torres, y otros, 2013), para ello en la presente investigación se utilizaron solventes como metanol, etanol y cloroformo para la extracción de aceite aplicando el método Bligh \& Dyer, del mismo modo en anteriores investigaciones también se reportaron estudios de producción de biocombustibles (biodiesel, hidrógeno, etanol y gas) a partir de microalgas, pero se realizaron en escalas de laboratorio o piloto. En 2008, PetroSun entregó información de su planta comercial de algas para la producción de biodiesel que tenía una capacidad de producir 4.4 millones de galones de aceite de algas por año con una superficie de 1100 acres ubicada en Río Hondo, Texas (Chen, y otros, 2018). La presente investigación identifica el área en hectáreas (ha) implicadas para el cultivo de las microalgas en un sistema Race Way.

En relación a los recursos nutricionales, aparte de Agua, $\mathrm{CO} 2$ y luz solar, fósforo y nitrógeno presentes como dos Principales aportes de nutrientes para el crecimiento de microalgas, junto con los macroelementos como N, P, Na, $\mathrm{Mg}, \mathrm{K}$ y Ca, se requiere de microelementos como $\mathrm{Mn}, \mathrm{B}$, $\mathrm{Mo}, \mathrm{Zn}$, Co y Fe que también son necesarios (Zhu, y otros, 2017), los medios BBM (Basal Bold Medium) y el CHU fueron utilizados en la presente investigación como aporte nutricional para el desarrollo de biomasa microalgal.

El objetivo de la investigación fue identificar el desempeño de la biomasa microalgal para la microproducción de biodiesel a partir de una brecha de potencial energético utilizando como modelo de estudio el fundo de la Universidad Católica de Santa María ubicado en Huasacache-Arequipa y la microempresa ALDECO.

\section{MATERIALES Y MÉTODOS}

\section{Cultivo de microalgas}

Se adquirieron $y$ se recolectaron microalgas, posteriormente se procedió a su purificación mediante diluciones seriadas en medio de cultivo líquido y repiques en placas de medio sólido BBM, para los escalamientos se realizaron pruebas preliminares con el objetivo de seleccionar la cepa más apta en condiciones ambientales y con mejor rendimiento lipídico.

Para el cultivo de las microalgas purificadas se utilizaron los medios BBM (Basal Bold Medium) y el CHU. La composición de dichos medios se muestra a continuación.
Tabla 1: Medios de cultivo especiales para Microalgas

\begin{tabular}{|c|c|c|}
\hline Nutrientes & $\mathrm{CHU}$ & BBM \\
\hline \multicolumn{3}{|c|}{ Macronutrientes (g/l) } \\
\hline $\mathrm{KH} 2 \mathrm{PO} 4$ & 0.5 & 175 \\
\hline $\mathrm{CaCl} 2$ & 0.04 & 25 \\
\hline $\mathrm{MgSO} 4$ & 0.2 & 75 \\
\hline $\mathrm{NaCl}$ & 1 & 25 \\
\hline $\mathrm{NaNO} 3$ & 2.5 & 250 \\
\hline $\mathrm{K} 2 \mathrm{HPO} 4$ & - & 75 \\
\hline $\mathrm{H} 3 \mathrm{BO} 3$ & - & 11.42 \\
\hline \multicolumn{3}{|c|}{ Micronutrientes (g/l) } \\
\hline $\mathrm{ZnSO} 4$ & 0.222 & 8.82 \\
\hline $\mathrm{CuSO} 4$ & 0.079 & 1.57 \\
\hline $\mathrm{H} 3 \mathrm{BO} 3$ & 2.86 & 11.42 \\
\hline $\mathrm{MnCl} 2$ & 1.81 & 1.44 \\
\hline $\mathrm{Na} 2 \mathrm{MoO} 4$ & 0.018 & 0.39 \\
\hline $\mathrm{Co}(\mathrm{NO} 3) 2$ & - & 0.49 \\
\hline \multicolumn{3}{|c|}{ Solución de Hierro (g/l) } \\
\hline Ácido Cítrico & 0.08 & - \\
\hline $\mathrm{FeSO} 4$ & 16.8 & - \\
\hline \multicolumn{3}{|c|}{ Solución $1(\mathrm{~g} / \mathrm{l})$} \\
\hline Na2EDTA & - & 50 \\
\hline $\mathrm{KOH}$ & - & 3.1 \\
\hline \multicolumn{3}{|c|}{ Solución $2(\mathrm{~g} / \mathrm{l})$} \\
\hline $\mathrm{FeSO} 4$ & - & 4.98 \\
\hline H2 SO4 (95-98\%) & - & $1 \mathrm{ml}$ \\
\hline
\end{tabular}

Se obtuvieron microalgas del entorno de la ciudad de estudio (Arequipa), para lo cual se esperó que se adaptaran a condiciones ambientales locales, el cultivo fue evaluado y sometido a escalamiento bajo las especificaciones que a continuación se mencionan.

Inicialmente se empezó con un inoculo de $20 \mathrm{ml}$, se procedió a cultivar en un matraz de $250 \mathrm{ml}$. Al llegar a la fase logarítmica tardía (7 días), se procedió a escalar el cultivo a un recipiente de $1000 \mathrm{ml}$ utilizando todo el inoculo del cultivo anterior, Se cultivó el alga por 7 días para alcanzar la fase logarítmica tardía. Finalmente utilizando el contenido total del recipiente de $1000 \mathrm{ml}$ se procedió a sembrar en recipientes de $5000 \mathrm{ml}$.

La cepa utilizada para escalamiento en piloto fue seleccionada bajo el criterio de contenido lipídico, para ello se utilizó pruebas de extracción de lípidos con el Método Bligh \& Dyer y el Método de extracción con hexano, los procedimientos se detallan a continuación

\section{Protocolo del método Bligh \& Dyer aplicado a la extracción de lípidos de microalgas}

Se pesó $0.2 \mathrm{~g}$ de biomasa seca, luego en un pomo se agregó la biomasa y los solventes: $1.6 \mathrm{ml}$ de metanol y 0.8 $\mathrm{ml}$ de cloroformo; se agitó por 15 horas (overnight). Al día siguiente, se agregó $0.8 \mathrm{ml}$ de cloroformo y se agitó durante 2 minutos. Se agregó $0.8 \mathrm{ml}$ de agua destilada y se volvió agitar por 2 minutos.

Luego se centrifugó a $3400 \mathrm{rpm}$ por un periodo de 15 
minutos. Se extrajo la capa superior (metanol-agua) contenida en los tubos de centrifuga con la ayuda de una pipeta Pasteur. Se filtró por gravedad y se lavó el tubo centrifuga con 0.8 $\mathrm{ml}$ de cloroformo, finalmente se dejó volatilizar el extracto y la biomasa residual. Se cuantificó los lípidos por medio de gravimetría.

\section{Protocolo del método con hexano (Ramírez Fajardo et al 2007)}

Se pesó $0.2 \mathrm{~g}$ de biomasa seca y se agregó $1 \mathrm{ml}$ de etanol, la mezcla fue agitada por 15 horas (overnight). Se separó la fase solida de la liquida por filtración. Se agregó a la fase solida $1 \mathrm{ml}$ de etanol y se agito por 1 hora a 500 rpm. Se separó por filtración y se combinó las 2 fases liquidas. Se agregó $2.75 \mathrm{ml}$ de agua destilada para ajustar la fase alcohólica a $40 \%$ de pureza. Luego se agregó $0.4 \mathrm{ml}$ de hexano, para permitir la migración de lípidos. Se separó la fase hexanica de la hidroalcoholica. La adición de $0.4 \mathrm{ml}$ de hexano con fase hidroalcoholica se repitió por 4 veces. Se combinó las fases hexanicas y se dejó evaporar el hexano. Se cuantifico los lípidos por gravimetría.

Se determinó el rendimiento de lípidos según la siguiente formula:

$$
\% \text { Lipidos }_{x}=\frac{L_{X}}{M_{x}} * 100 \%
$$

Donde $\mathrm{M} \_\mathrm{x}$ representa la biomasa seca inicial y L_x la cantidad de lípidos obtenidos luego de la extracción con el método Bligh \& Dyer o el método con Hexano (Ramírez Fajardo).

\section{Implementación de piscinas tipo Raceway}

Para el escalamiento se utilizaron pozas experimentales diseñadas con las siguientes especificaciones, un largo total de $2.44 \mathrm{~m}$ y un ancho de $0.44 \mathrm{~m}$, el alto total es de 0.40 $\mathrm{m}$ para un alto efectivo (profundidad máxima del agua) de $0.3 \mathrm{~m}$, el volumen total es de $0.41 \mathrm{~m} 3$ lo cual permite un volumen efectivo de $0.31 \mathrm{~m} 3$, los materiales utilizados es la Geomembrana blanca de $1 \mathrm{~mm}$ de espesor poliolefina fortificada (Enviroliner $6000 \mathrm{HD}$ ) especial para acuicultura y crianza de algas, el marco exterior de apoyo en platina y tubos metálicos.

El sistema utilizó 3 ejes de 8 paletas cada una, giro de 100 r.p.m. que permite alcanzar velocidades del agua de 50 $\mathrm{cm} /$ segundo (flujo laminar), en la figura 1 se puede apreciar las diferentes vistas del sistema.

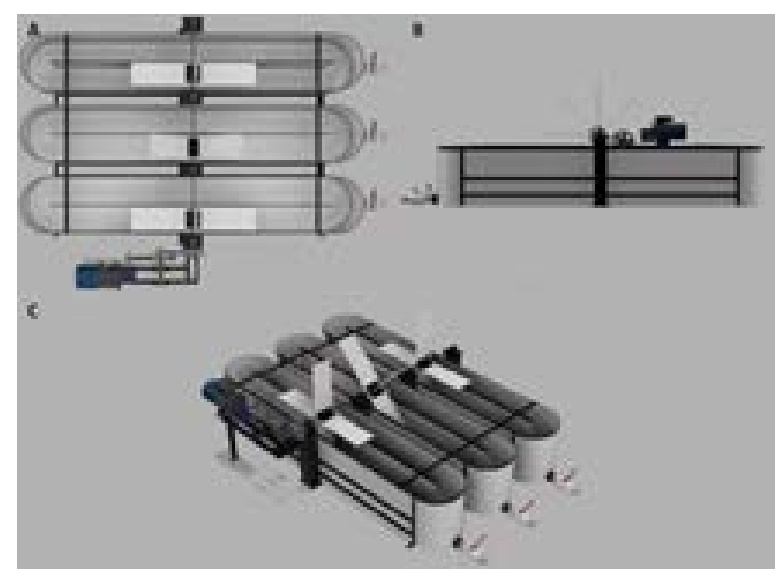

Fig. 1: Reactor experimental tipo Raceway: en la imagen se observan las diferentes vistas del reactor, A) Vista aérea donde se puede apreciar el flujo del sistema, B) Vista vistas del reactor, A) Vista aérea donde se puede apreciar el flujo del sistema, B) Vista
frontal donde se observa el sistema de poleas y motor, C) Vista 3D donde se aprecia el frontal donde se observa el sistema de poleas y motor, C) Vista 3D donde se aprecia el
diseño completo del sistema, D) Vista real del sistema implementado y funcional en el diseño completo del sistema, D)
fundo Huasacache de la UCSM.

\section{Cultivo de microalgas a escala piloto}

Se utilizaron los cultivos de $5 \mathrm{~L}$ para escalarlos a $20 \mathrm{~L}$ con el medio BBM durante un periodo de 7 días, se agregaron $20 \mathrm{~L}$ de inóculo a cada una de las pozas Raceway a un nivel de $300 \mathrm{~L}$, posterior se esperó la generación de biomasa durante un periodo de 7 días, la biomasa se recolecto agregando sulfato de aluminio como coadyuvante para su sedimentación, se eliminó el líquido sobrenadante procurando no perder biomasa, a continuación se filtró la biomasa en tela para evitar recolectar contaminantes, el precipitado se llevó a laboratorio para ser concentrada y secada.

\section{Procedimiento para la obtención de biodiesel}

Para la obtención de biodiesel se trabajó a partir de biomasa seca, el aceite se extrajo con hexano (C6H14) y la transesterificación se realizó utilizando $5 \mathrm{~g}$ del aceite extraído y $\mathrm{MeOH}$ en relación 6:1 molar con $1.4 \mathrm{~mL}$ de THF (Tetrahidrofurano) y $0.24 \mathrm{~g}$ de $\mathrm{AlCl} 3$.

\section{Determinación del Potencial Bioenergético}

La metodología utilizada para la determinación del potencial energético de la biomasa de microalgas cultivadas en sistemas Race Way son descritos a continuación:

El Potencial de biomasa disponible (PSD) consistió en determinar la disponibilidad de biomasa seca por metro cuadrado acorde a los resultados obtenidos de la cosecha de microalgas en los reactores pilotos raceway, en relación al aceite obtenido el Potencial del Combustible Generado (PCG) se determinó acorde a los ensayos trabajados en laboratorio y su rendimiento en relación a la cantidad de aceite obtenido.

El Potencial Factible de producción (PFP) se identificó mediante entrevistas realizadas a microempresarios los cuales brindaron los parámetros para el escalamiento productivo acorde a las condiciones experimentales propuestas y a partir del PE identificado en galones producidos. 


\section{RESULTADOS Y DISCUSIONES}

\section{Cultivo de microalgas}

Entre las microalgas adquiridas (Scenedesmus sp., Chlorella sp., Pediastrum sp. y haematococcus sp.) la cepa de Haematococcus sp. fue la única que prolifero en medio BBM obteniendo $0.21 \mathrm{~g} / \mathrm{L}$ de biomasa, de las microalgas recolectadas en el entorno de Arequipa las que proliferaron en medio CHU y BBM fueron Chlorella sp. (CHU: $2.13 \mathrm{~g} / \mathrm{L}$ y BBM $2.78 \mathrm{~g} / \mathrm{L}$ ) y Scenedesmus sp. $0.35 \mathrm{~g} / \mathrm{L}$, las cepas fueron trabajadas en módulos experimentales con aireación y una intensidad de luz blanca de $540 \mathrm{umol} / \mathrm{m}^{2} \mathrm{~s}$, en comparación con otros resultados obtenidos los rendimientos fueron menores, por ejemplo se encontró que un cultivo mixotrófico con intensidad de luz $170 \mathrm{uE} / \mathrm{m}^{2} \mathrm{~s}$ a una concentración de acetato $30 \mathrm{mM}$ generó $0,243 \mathrm{~g} / \mathrm{L}$ de biomasa (Chul Jeon et al., 2006), para el cultivo de Chlorella sp. se encontró un rendimiento de $3.72 \mathrm{~g} / \mathrm{L}$ de biomasa en medio de acetato y 2.17 en medio con carbonato (González-Delgado y otros, 2017) y para Scenedesmus sp. se encontró reportes de 0,243 g/L de biomasa utilizando NaHCO3 (Quevedo, 2007)(Tabla 2).

Tabla 2: Generación de biomasa microalgal en medio CHU y BBM

\begin{tabular}{|c|c|c|c|}
\hline \multirow{2}{*}{ Microalga } & \multirow{2}{*}{ Condiciones } & \multicolumn{2}{|c|}{ Biomasa, $\mathrm{g} / \mathrm{L}$} \\
\hline & & $\mathrm{CHU}$ & BBM \\
\hline Chlorella sp. & Centrifugado y secado & 2.00 & - \\
\hline Chlorella sp. & Centrifugado y secado & - & 2.13 \\
\hline Scenedesmus sp & Filtrado y secado & - & 0.35 \\
\hline Chlorella sp. & $\begin{array}{l}\text { Precipitado con sulfato de } \\
\text { aluminio, centrifugado y } \\
\text { secado }\end{array}$ & - & 2.30 \\
\hline Haematococcus sp. & $\begin{array}{l}\text { Precipitado con sulfato de } \\
\text { aluminio, centrifugado y } \\
\text { secado }\end{array}$ & - & 0.39 \\
\hline
\end{tabular}

Los resultados preliminares obtenidos para la extracción de lípidos aplicando el método Bligh \& Dyer sobre la biomasa seca de Chlorella sp. se obtuvo un rendimiento de $6.83 \%$ a partir de $0.0138 \mathrm{~g}$ de lípido extraído (Fórmula 1 ), del mismo modo aplicando el método con hexano se obtuvo un rendimiento de $7.49 \%$ a partir de $0.0151 \mathrm{~g}$ de lípido extraído (Tabla 3).
Tabla 3: Generación de lípidos a partir de biomasa microalgal.

\begin{tabular}{|c|c|c|c|c|}
\hline Microalga & Método & Condiciones & Biomasa, $\mathrm{g}$ & Lípido, $\%$ \\
\hline Chlorella sp. & Bligh \& Dyer & $\begin{array}{c}\text { Tratamiento con metanol } \\
\text { y cloroformo y separación } \\
\text { por centrifugación y } \\
\text { filtrado por gravedad }\end{array}$ & 0.202 & 6.83 \\
\hline Chlorella sp. & $\begin{array}{l}\text { Método con } \\
\text { hexano }\end{array}$ & $\begin{array}{l}\text { Tratamiento con etanol en } \\
\text { agitación y estabilizado a } \\
40 \% \text {, agregado de hexano } \\
\text { por cuatro repeticiones } \\
\text { para migrar lípidos } \\
\text { y cuantificación por } \\
\text { gravimetría }\end{array}$ & 0.202 & 7.49 \\
\hline Chlorella sp. & & $\begin{array}{l}\text { Pretratamiento con sulfato } \\
\text { de aluminio y filtración }\end{array}$ & 0.460 & 8.33 \\
\hline Haematococcus sp. & $\begin{array}{l}\text { Extraccion en } \\
\text { Soxhlet con } \\
\text { hexano }\end{array}$ & $\begin{array}{l}\text { por gravedad, maceración } \\
\text { con hexano durante } 7 \text { días }\end{array}$ & 0.077 & 4.28 \\
\hline Scenedesmus sp & & $\begin{array}{l}\text { y extraccion con Soxhlet } \\
\text { durante } 30 \mathrm{~min} \text { a } 70^{\circ} \mathrm{C}\end{array}$ & 0.200 & 17.12 \\
\hline
\end{tabular}

Se encontró que la microalga Scenedesmus sp genera mayor contenido de lípidos, sin embargo en relación a la generación de biomasa es 6.57 veces menor a la generación de biomasa de Chlorella sp. que para fines de escalamiento implicaría mayor área de cultivo representado mayor uso de recurso durante un periodo de 7 días.

Se eligió a la microalga Chlorella como principal fuente de lípido para la obtención de biodiesel.

\section{Resultados del escalamiento a piloto}

Se cosechó 1977,67 g de biomasa húmeda a partir de 300L de medio, se trabajó por triplicado durante tres jornadas, la primera jornada se preparó el cultivo a un volumen de $100 \mathrm{~L}$ con un inóculo de $20 \mathrm{~L}$, la segunda jornada se nivelo el volumen a $300 \mathrm{~L}$ y la tercera jornada se aplicó sulfato de aluminio para la cosecha, las dos primeras jornadas se trabajaron durante un periodo de 7 días, la tercera jornada se trabajó en un día.

De la biomasa húmeda cosechada se obtuvo un promedio de 246,00 g de biomasa seca con un porcentaje promedio de rendimiento del $12.44 \%$ con una generación de $0.82 \mathrm{~g} / \mathrm{L}$ (Tabla 4).

Tabla 4: Obtención de biomasa seca.

\begin{tabular}{ccccc}
\hline $\begin{array}{c}\text { Obtención de } \\
\text { biomasa seca }\end{array}$ & Muestra 1 & Muestra 2 & Muestra 3 & Promedio \\
\hline $\begin{array}{c}\text { Biomasa húmeda, } \\
\text { g. }\end{array}$ & 1950,00 & 2010,00 & 1973,00 & 1977,67 \\
$\begin{array}{c}\text { Biomasa seca, g. } \\
245,00\end{array}$ & 251,00 & 242,00 & 246,00 \\
$\begin{array}{c}\text { Biomasa seca, \%. } \\
\text { Generación de } \\
\text { biomasa, g/L. }\end{array}$ & $0,82,56$ & 12,49 & 12,27 & 12,44 \\
\hline
\end{tabular}

\section{Obtención de biodiesel}

\section{Extracción de aceite}

Se encontró que a partir de un promedio de biomasa seca de $10,03 \mathrm{~g}$ se logró obtener un promedio de $0,62 \mathrm{~g}$ con un rendimiento del $6.17 \%$ y una generación de aceite de 614,90 g (Tabla 4), en otras investigaciones la productividad lipídica de la cepa AVFF007 fue de $46.3 \mathrm{mg}$ / L / día, lo que permite comparar con las productividades lipídicas de otras especies de microalgas (Muto, y otros, 2017). 
Tabla 5: Extracción de aceite a partir de biomasa seca

\begin{tabular}{ccccc}
\hline Extracción del aceite & Muestra 1 & Muestra 2 & Muestra 3 & Promedio \\
\hline Muestra microalga biomasa & 10,03 & 10,02 & 10,05 & 10,03 \\
Aceite extraído, g. & 0,62 & 0,61 & 0,62 & 0,62 \\
Aceite extraído, \%. & 6,21 & 6,14 & 6,16 & 6,17 \\
Generación de aceite, g/Kg. & 619,17 & 612,62 & 612,92 & 614,90 \\
\hline
\end{tabular}

\section{Transesterificación}

Se obtuvo un promedio de un $55.60 \%$ de aceite transesterificado (biodiesel) a partir de $0.78 \mathrm{~g}$ de aceite inicial resultando en un rendimiento de 554,32 $\mathrm{g} / \mathrm{Kg}$ de aceite transesterificado (Tabla 6).

Tabla 6: Trasesterificación de aceite obtenido de biomasa seca.

\begin{tabular}{ccccc}
\hline Transesterificación & Muestra 1 & Muestra 2 & Muestra 3 & Promedio \\
\hline Muestra aceite inicial(g) & 0,78 & 0,79 & 0,78 & 0,78 \\
$\begin{array}{c}\text { Muestra aceite } \\
\text { transterificado (g) }\end{array}$ & 0,43 & 0,45 & 0,44 & 0,44 \\
$\begin{array}{c}\text { Aceite transterificado } \\
(\%)\end{array}$ & 55,60 & 57,24 & 56,43 & 56,43 \\
$\begin{array}{c}\text { Generación de } \\
\text { biodiesel, g/Kg. }\end{array}$ & 556,03 & 572,42 & 564,34 & 564,32 \\
\hline
\end{tabular}

\section{Resultados Generales}

La tabla 7 muestra los resultados de la etapa de obtención de biodiesel; se identificó que el porcentaje de biomasa seca obtenido fue de 12.44+/-0.38 con una desviación estándar de 0.1513 , el porcentaje de aceite extraído a partir de la biomasa seca fue de $6.17+/-0.09$ y el porcentaje de aceite esterificado obtenido a partir del total de aceite extraído fue de $56.45+/$ 2.04 , en trabajos de investigación relacionados se encontró brechas de contenido de FAME en transesterificación de $64.98 \pm 2.88 \%$ en biodiesel crudo (Torres, Acien, GarcíaCuadra, \& Navia, 2017).

Tabla 7: Estadística descriptiva para cada etapa en la obtención de Biodiesel a partir de biomasa seca.

\begin{tabular}{cccc}
\hline Muestras & $\begin{array}{c}\text { Biomasa seca } \\
(\%)\end{array}$ & $\begin{array}{c}\text { Aceite extraído } \\
(\%)\end{array}$ & $\begin{array}{c}\text { Aceite } \\
\text { transterificado } \\
(\%)\end{array}$ \\
\hline Muestra 1 & 12.56 & 6.21 & 55.6 \\
Muestra 2 & 12.49 & 6.14 & 57.24 \\
Muestra 3 & 12.27 & 6.16 & 56.43 \\
Promedio & $12.44+/-0.38$ & $6.17+/-0.090$ & $56.45+/-2.04$ \\
Desviación estándar & 0.1513 & 0.0361 & 0.8200 \\
Varianza & 0.0229 & 0.0013 & 0.6724 \\
CV (\%) & 1.22 & 0.58 & 1.45 \\
\hline
\end{tabular}

\section{Potencial energético}

La biomasa generada del reactor piloto es de $246 \mathrm{~g}$ de biomasa seca por poza de cultivo de $300 \mathrm{~L}$ cada una, se cultivaron en tres 3 pozas piloto experimentales haciendo un total de $738 \mathrm{~g}$ a partir de un volumen de $900 \mathrm{~L}$ de cultivo, como resultado el potencial de biomasa disponible (PBD) es de $820 \mathrm{~g} / \mathrm{m} 3$, el rendimiento de aceite extraído es del 6.17 $\%$ pudiendo obtener $50,59 \mathrm{~g} / \mathrm{m} 3$ de la materia prima para la obtención de aceite Transesterificado (biodiesel), se obtuvo como resultado un potencial de combustible generado (PCG) de $28,55 \mathrm{~g} / \mathrm{m} 3$ de cultivo.

De acuerdo al ministerio de la producción en el Perú el uso potencial del biodiesel se encuentra en la mezcla con carburantes con un contenido del 5\% de biodiesel, no se encontró una categorización como microproductores en base a la cantidad de combustible generado, por tal motivo el potencial factible de producción de biodiesel (PFP) se determinó en base a entrevistas realizadas con microproductores ellos informan que generaban un promedio de 40 galones semanales, sin embargo tenían una capacidad de producción de 200 galones diarios (BIOCUSCO, 2018), pero debido a la baja disponibilidad de aceite de sus principales proveedores como restaurantes y hoteles no lograban cubrir su capacidad ociosa, uno de los microproductores que generaba 50 galones mensuales en Arequipa informó que el principal riesgo en su modelo de negocio fue la baja del costo de petróleo (ALDECO, 2018) lo cual afectó su flujo de caja, ellos indican que son considerados pequeños productores los que tienen la capacidad de proveer a la industria de carburantes para la mezcla con el combustible y que el principal factor fue el costo de análisis del Biodiesel al hacer la entrega del producto con un valor de $200 \$$, para tal motivo el modelo de negocio de los microproductores radicaba en proveer a pequeños productores que a la vez funcionaban como acopiadores.

Para determinar el PFP se utilizó el modelo de ALDECO ( $\mathrm{PFP}=50 \mathrm{gal} / \mathrm{mes}$ ), siendo la densidad del biodiesel $\mathrm{B} 100$ $0,88 \mathrm{~g} / \mathrm{cm} 3$, para ello siendo el PCG equivalente a $28,55 \mathrm{~g} / \mathrm{m} 3$ de cultivo el volumen de $\mathrm{B} 100$ es $0,0071 \mathrm{gal} / \mathrm{m} 3$ de medio de cultivo BBM utilizado, acorde a los resultados se requeriría un volumen de 7042,25 m3 de medio de cultivo, teniendo en cuenta que la altura para el cultivo recomendable para microalgas es de $0,30 \mathrm{~cm}$ implicaría tener un espacio de 2,35 ha dedicado sólo a cultivo de microalgas.

Siendo el poder calorífico del biodiesel $40 \mathrm{MJ} \mathrm{kg}-1$, el potencial energético (PE) de 50 gal B100 es de 8042,2495 $\mathrm{MJ} \mathrm{Kg-1} \mathrm{por} \mathrm{cada} \mathrm{mes.}$

Otros trabajos de investigación evalúan Este estudio realiza una evaluación técnico-económica ambiental (ETEA) para múltiples biorrefinerías de microalgas en diferentes lugares como Bélgica e India. La metodología ETEA, que integra aspectos de las metodologías TEA y LCA y proporciona un marco claro para un modelo de evaluación integrado, ha sido propuesto y discutido. El escenario en India tiene una mayor rentabilidad con un VAN de $€ 40$ millones en un período De 10 años, mientras que el impacto ambiental en Bélgica es menor. La inclusión de un paso de reciclaje medio. Proporciona el mejor escenario desde ambas perspectivas. 
La identificación de estos parámetros por la ETEA guía los desarrollos tecnológicos futuros y acorta el tiempo de comercialización de las biorrefinerías basadas en microalgas (Thomassen, Van Dael, \& Van Passel, 2018).

\section{CONCLUSIONES}

La presente investigación demostró que es posible obtener $0,82 \mathrm{~g} / \mathrm{L}$ de biomasa microalgal en un sistema Raceway logrando extraer $614,90 \mathrm{~g} / \mathrm{kg}$ de aceite a partir de biomasa microalgal seca alcanzando a generar 554,32 g/Kg de aceite transesterificado (Biodiesel- B100), los resultados determinaron que para un microproductor que busca alcanzar un potencial energético (PE) de 8042,2495 MJ Kg-1 a partir de un potencial de producción factible (PPF) de $50 \mathrm{gal} / \mathrm{mes}$ implica destinar un área de 2,35 ha bajo un rendimiento determinado por el potencial de biomasa disponible (PBD) de $820 \mathrm{~g} / \mathrm{m} 3$ y de combustible generado (PCG) de $28,55 \mathrm{~g} / \mathrm{m} 3$ en medio de cultivo BBM.

\section{REFERENCIAS BIBLIOGRÁFICAS}

1. Chen, J., Li, J., Dong, W., Zhang, X., Tyagi, R., Drogui, P., \& Surampalli, R. (2018). The potential of microalgae in biodiesel production. Renewable and Sustainable Energy Reviews, 90, 336-346. Renewable and Sustainable Energy Reviews, 90, 336-346.

2. de Luna, M., Doliente, L., Ido, L., \& Chung, T. (2017). In situ transesterification of Chlorella sp. microalgae using LiOH-pumice catalyst. Journal of environmental chemical engineering, 5(3), 2830-2835.

3. Ghosh, S., Roy, S., \& Das, D. (2015). Improvement of biomass production by Chlorella sp. MJ 11/11 for use as a feedstock for biodiesel, Appl. Biochem. Biotechnol. 175 (2015). Appl. Biochem. Biotechnol, 175(3322-3335).

4. Hanifzadeh, M., Sarrafzadeh, M. H., M., Nabati Z, Z., Tavakoli, O., \& Feyzizarnagh, H. (2018). Technical, economic and energy assessment of an alternative strategy for mass production of biomass and lipid from microalgae. Journal of environmental chemical engineering, 6(1), 866-873.

5. Muto, M., Nojima, D., Yue, L, L., Kanehara, H., Naruse, H., Ujiro, A., \& Tanaka, T. (2017). Potential of water surface-floating microalgae for biodiesel production: Floating-biomass and lipid productivities. Journal of bioscience and bioengineering, 123(3), 314-318.

6. Sánchez, E., Ojeda, K., El-Halwagi, M., \& Kafarov. (2011). Biodiesel from microalgae oil production in two sequential esterification/transesterification reactors: Pinch analysis of heat integration. Chemical engineering journal, 176, 211-216.

7. Thomassen, G., Van Dael, M., \& Van Passel, S. (2018). The potential of microalgae biorefineries in Belgium and India: An environmental techno-economic assessment. Bioresource technology, 267, 271-280.

8. Torres, C., Ríos, S., Torras, C., Salvadó, J., Mateo-Sanz, J., \& Jiménez, L. (2013). Microalgae-based biodiesel: a multicriteria analysis of the production process using realistic scenarios. Bioresource technology, 147, 7-16.

9. Torres, S., Acien, G., García-Cuadra, F., \& Navia, R. (2017). Direct transesterification of microalgae biomass and biodiesel refining with vacuum distillation. Algal Research, 28, 30-38.

10. Zhang, X., Yan, S., Tyagi, R., \& Surampalli, R. (2013). Biodiesel production from heterotrophic microalgae through transesterification and nanotechnology application in the production. Renewable and Sustainable Energy Reviews, 26, 216-223.

11. Zhu, L., Nugroho, Y., Shakeel, S., Li, Z., Martinkauppi, B., \& Hiltunen, E. (2017). Using microalgae to produce liquid transportation biodiesel: what is next? Renewable and Sustainable Energy Reviews,, 78, 391-400. 